\title{
Renovascular Hypertension Due to Midaortic Syndrome Associated with Chronic Takayasu Arteritis Successfully Treated with Multiple Simultaneous Visceral Bypasses
}

\author{
Hyokee Kim ${ }^{1}$ and Ahram Han ${ }^{2}$ \\ ${ }^{1}$ Department of Surgery, Korea University Guro Hospital, Seoul, ${ }^{2}$ Department of Surgery, Seoul National University Hospital, Seoul, \\ Korea
}

A 52-year-old female was transferred with a 2-year history of intermittent claudication and high blood pressure (BP). She was treated for rheumatoid endocarditis in childhood. The BPs of the left arm and right arm were 187/99 and 169/90 mmHg, respectively. Carotid bruit was absent

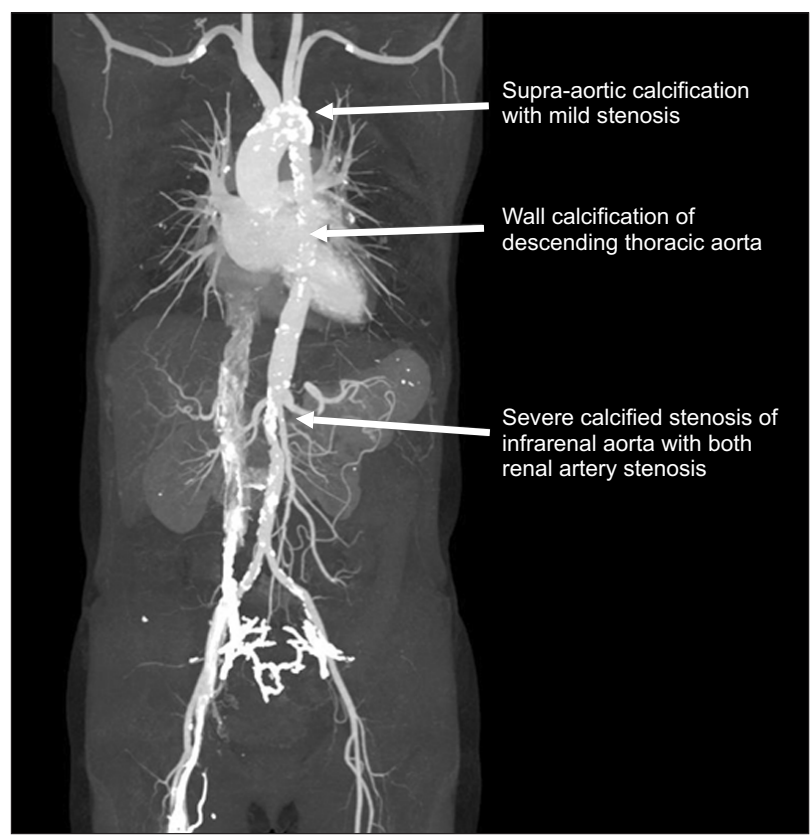

Fig. 1. Preoperative computed tomography angiography showed multiple calcification and stenosis in the aorta. and the pulses of all four extremities were palpable. Blood tests including inflammatory markers were unremarkable. Computed tomography showed tight segmental narrowing of the abdominal aorta, severe stenosis of the bilateral renal

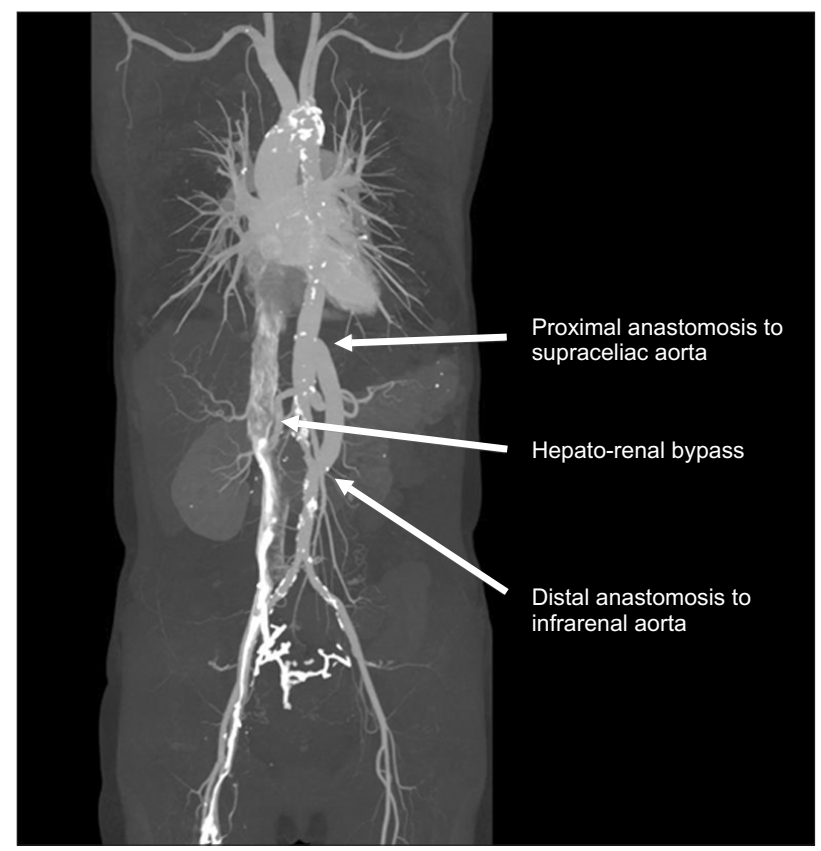

Fig. 2. Postoperative computed tomography angiography showed supraceliac aortic-infrarenal aortic bypass and hepato-renal bypass.

Received December 20, 2021, Accepted December 23, 2021, Published on December 31, 2021

Corresponding author: Ahram Han, Division of Vascular Surgery, Biomedical Research Institute, Seoul National University Hospital, 101 Daehak-ro, Jongno-gu, Seoul 03080, Korea

Tel: 82-2-2072-0297, Fax: 82-2-766-3975, E-mail: 66007@snuh.org, https://orcid.org/0000-0002-3866-5214

Copyright (c) 2021 The Korean Society for Vascular Surgery

This is an Open Access article distributed under the terms of the Creative Commons Attribution Non-Commercial License (http://creativecommons.org/licenses/by-nc/4.0) which permits unrestricted non-commercial use, distribution, and reproduction in any medium, provided the original work is properly cited.

Cite this article; Vasc Specialist Int 2021. https://doi.org/10.5758/vsi.210800 


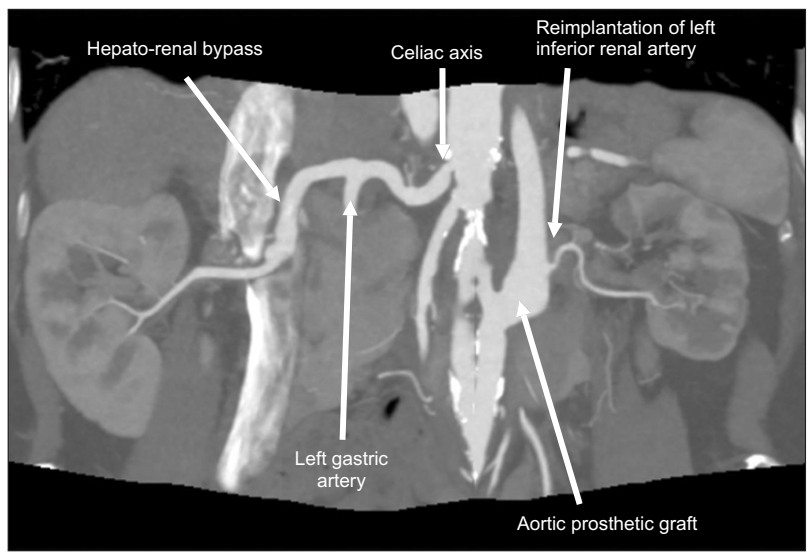

Fig. 3. Reconstructed computed tomography angiography showed the both renal artery revascularization; right hepato-renal bypass and left inferior renal artery reimplantation.

arteries (RAs), diffuse calcification of the thoracic aorta, and supra-aortic arteries compatible with chronic Takayasu arteritis (TAK) (Fig. 1). Rheumatologists confirmed a lack of active vasculitis. An elective operation was performed consisting of a supraceliac aortic-infrarenal aortic bypass with a 14-mm Dacron graft, left inferior RA reimplantation to the graft, and hepato-right renal bypass with a reversed great saphenous vein (Fig. 2, 3). The postoperative course was uneventful and her BP was well controlled with oral nifedipine. After a year of aspirin and clopidogrel, the patient was maintained on aspirin alone. At 2 years of followup, there is no evidence of graft stenosis or renal dysfunction.

Mid-aortic syndrome (MAS) is a rare disease characterized by severe stenosis of the distal thoracic or abdominal aorta with frequent involvement of visceral and renal arteries [1]. TAK is one of the most common causes of MAS in adults. MAS commonly presents with claudication and uncontrolled hypertension related to aortic coarctation or RA stenosis. The possibility of surgical revascularization depends on the extent and location of the aortic disease and calcification. In this case, proximal anastomosis was performed on the supraceliac aorta, where the calcification was the least evident. While the left inferior RA was reimplanted to the graft at 5 o'clock position, the right RA stenosis was corrected by a different inflow: hepato-renal bypass using a vein graft. Evaluation and control of disease activity are of paramount importance upon vascular reconstructions in TAK [2-4]. Fortunately, there was no evidence of recurrent vasculitis in this case.

\section{REFERENCES}

1) Kim SM, Jung IM, Han A, Min Sl, Lee $\mathrm{T}$, Ha J, et al. Surgical treatment of middle aortic syndrome with Takayasu arteritis or midaortic dysplastic syndrome. Eur J Vasc Endovasc Surg 2015;50:206-212.

2) Min SK. Takayasu arteritis. J Korean
Soc Vasc Surg 2005;21:206-212.

3) Ahn MS, Kim MY, Huh S, Min SK, Ha J, Chung JK, et al. Surgical treatment of Takayasu arteritis. J Korean Soc Vasc Surg 2001;17:24-31.

4) Park YJ, Min SK, Ha J, Kim YS, Kim SJ. Surgical treatment of a suprarenal ab- dominal aortic pseudoaneurysm that recurred 20 years after aorto-renal bypass in a patient with Takayasu arteritis. J Korean Soc Vasc Surg 2008;24:56-59. 